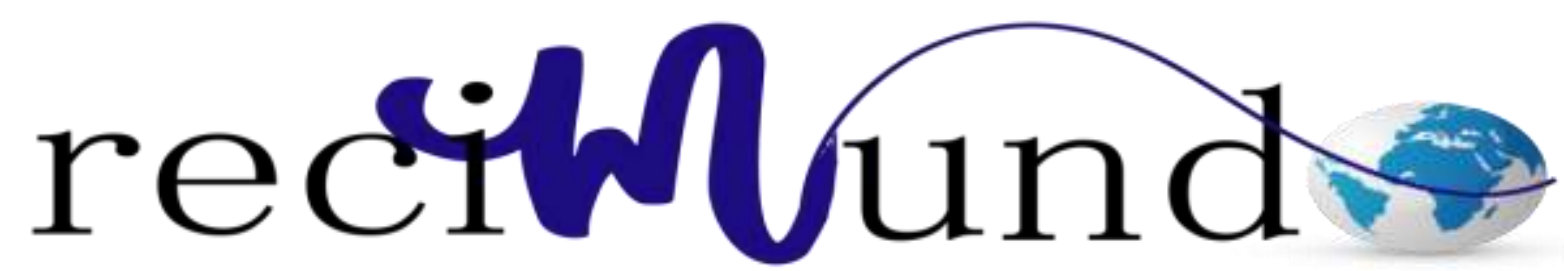

Revista Científica Mundo de la Investigación y el Conocimiento

$$
\text { María Isabel Pacheco Arias a ; Holguer Estuardo Romero Urrea }{ }^{\text {b }}
$$

Perfil lipídico como factor de riesgo cardiovascular en militares activos. Hospital General II-de Libertad 2019

Lipid profile as a cardiovascular risk factor in active military General Hospital IIde Libertad 2019

Revista Científica Mundo de la Investigación y el Conocimiento. Vol. 3 núm.3. Esp., noviembre, ISSN: 2588-073X, 2019, pp. 438-459

DOI: $10.26820 /$ recimundo/3.(3.Esp).noviembre.2019.438-459

URL: http://recimundo.com/index.php/es/article/view/611

Código UNESCO: 3205 Medicina Interna

Tipo de Investigación: Artículo de Revisión

(C) RECIMUNDO; Editorial Saberes del Conocimiento, 2019

Recibido: 15/09/2019

Aceptado: 23/10/2019

Publicado: 30/11/2019

Correspondencia: mar.isabel.87@ hotmail.com

a. Médico; Investigadora Independiente; Guayaquil, Ecuador; mar.isabel.87@ hotmail.com

b. Diploma Superior en Gestión de Desarrollo de los Servicios de Salud; Magister en Gerencia de Servicios de Salud; Doctor en Ciencias de la Salud; Master en Salud Mental Itinerario: Clínica Social, la Práctica Clínica de los Conflictos Individuales y Sociales en el Mundo Contemporáneo; Doctor en Psicología Clínica; Universidad Estatal de Milagro; Guayaquil, Ecuador; hromerou@unemi.edu.ec 


\section{Perfil lipídico como factor de riesgo cardiovascular en militares activos \\ Hospital General II-de Libertad 2019}

Vol. 3, núm. 3 Esp., (2019)

María Isabel Pacheco Arias; Holguer Estuardo Romero Urrea

\section{RESUMEN}

Las enfermedades cardiovasculares se han convertido en un problema de Salud Pública para todos los países del planeta. En este sentido, sabemos la importancia que tiene la prevención de este tipo de enfermedades, y uno de los métodos más populares y precisos es el estudio del perfil lipídico de los pacientes, de manera conjunta con el Índice de Masa Corporal y los niveles de glucosa en sangre. El desarrollo del perfil lipídico de los pacientes. Las enfermedades cardiovasculares (ECV) son una de las causas más comunes de muerte y discapacidad en el mundo, y en el Ecuador, representan la primera causa de muerte. Este trabajo de investigación, es de tipo cuantitativo, ya que considera las mediciones de los parámetros asociados al perfil lipídico, la tensión arterial, el índice de masa corporal y el nivel de glucosa en la sangre. Además, esta investigación, es considerada un trabajo de cohorte trasversal con base exploratoria y tipo descriptivo, ya que, con los datos obtenidos de las fichas citadas, se describirán los niveles de los elementos del perfil lipídico de la población estudiada, y las variables asociadas a la prevalencia del riesgo cardiovascular. Como conclusiones tenemos que del análisis de los datos se desprende que gran parte de la población estudiada padece sobrepeso. Está claro que la mayoría de los militares estudiados, presenta alteraciones en el perfil lipídico, centrándose principalmente en niveles elevados de triglicéridos, colesterol total, colesterol HDL y bajos niveles de colesterol LDL. La distribución de la variable género, refleja la mínima participación de las mujeres en la vida militar, todo esto, porque hasta hace pocos años, las mujeres no eran admitidas en ningún tipo de programa de formación militar y, además en ciertos estratos sociales, existe rechazo a las mujeres que optan por esta profesión.

Palabras clave: Militares; Dislipidemias; Colesterol; Arterial; Cardiovascular. 


\title{
Perfil lipídico como factor de riesgo cardiovascular en militares activos Hospital General II-de Libertad 2019
}

Vol. 3, núm. 3 Esp., (2019)

María Isabel Pacheco Arias; Holguer Estuardo Romero Urrea

\begin{abstract}
Cardiovascular diseases have become a Public Health problem for all countries of the planet. In this sense, we know the importance of preventing such diseases, and one of the most popular and accurate methods is the study of the lipid profile of patients, together with the Body Mass Index and glucose levels. in blood The development of the lipid profile of patients. Cardiovascular diseases (CVD) are one of the most common causes of death and disability in the world, and in Ecuador, they represent the leading cause of death. This research work is quantitative, since it considers the measurements of the parameters associated with the lipid profile, blood pressure, body mass index and blood glucose level. In addition, this research is considered a cross-sectional cohort work with an exploratory basis and descriptive type, since, with the data obtained from the aforementioned files, the levels of the elements of the lipid profile of the studied population will be described, and the associated variables to the prevalence of cardiovascular risk. As conclusions we have that the analysis of the data shows that a large part of the population studied is overweight. It is clear that the majority of the military studied, presents alterations in the lipid profile, focusing mainly on high levels of triglycerides, total cholesterol, HDL cholesterol and low levels of LDL cholesterol. The distribution of the gender variable reflects the minimum participation of women in military life, all this, because until a few years ago, women were not admitted to any type of military training program and, in addition to certain social strata, there is I reject women who choose this profession.
\end{abstract}

Keywords: Military; Dyslipidemias; Cholesterol; Arterial; Cardiovascular. 


\section{Perfil lipídico como factor de riesgo cardiovascular en militares activos \\ Hospital General II-de Libertad 2019}

Vol. 3, núm. 3 Esp., (2019)

María Isabel Pacheco Arias; Holguer Estuardo Romero Urrea

\section{Introducción.}

Las enfermedades cardiovasculares (ECV) son una de las causas más comunes de muerte y discapacidad en el mundo, y en el Ecuador, representan la primera causa de muerte (Organización Mundial de la Salud, 2017). Se sabe que cerca de 30\% de la población ecuatoriana, mayor de 40 años, padece algún tipo ECV. Estas enfermedades se producen por dos factores, uno es la genética, factor que no se puede cambiar. Y el otro, es el estilo de vida, que tiene que ver con la alimentación, el sedentarismo, el tabaquismo, el alcoholismo; elementos que causan graves desórdenes en el organismo y que pueden producir hipertensión, diabetes y arterioesclerosis, enfermedades que incrementan el riesgo de prevalencia de ECV.

Las enfermedades cardiovasculares se han convertido en un problema de Salud Pública para todos los países del planeta. Es así que, en Estados Unidos, el $53 \%$ de los adultos padecen alteraciones en la concentración sérica de lípidos. Todo esto porque varios estudios han determinado que, el $30 \%$ de los norteamericanos, vive con un elevado nivel de triglicéridos en sangre, y alrededor del $27 \%$ de estos ciudadanos, presentan altos niveles de LDL colesterol, además de que el $23 \%$ presenta bajos niveles HDL, considerado lípido protector.

En este sentido, sabemos la importancia que tiene la prevención de este tipo de enfermedades, y uno de los métodos más populares y precisos es el estudio del perfil lipídico de los pacientes, de manera conjunta con el Índice de Masa Corporal y los niveles de glucosa en sangre. El desarrollo del perfil lipídico de los pacientes, permite establecer parámetros de riesgo cardiovascular en función de las alteraciones de las lipoproteínas identificadas mediante el análisis clínico de la sangre del paciente (Estruch \& Camafort, 2015). 


\section{Perfil lipídico como factor de riesgo cardiovascular en militares activos Hospital General II-de Libertad 2019}

Vol. 3, núm. 3 Esp., (2019)

María Isabel Pacheco Arias; Holguer Estuardo Romero Urrea

La dislipidemia es un trastorno metabólico que se caracteriza por la alteración de los niveles séricos de las lipoproteínas, incluyendo al Colesterol Total (CT), Triglicéridos (TG), Lipoproteínas de Alta Densidad (HDL), y Lipoproteínas de baja densidad (LDL). Este trastorno aparece por diversos aspectos, que pueden ser genéticos, patológicos o ambientales. Las personas que lo padecen, llegan a aumentar su riesgo de desarrollar enfermedades crónicas no trasmisibles.

El conjunto de enfermedades que alteran la presencia de lípidos en el torrente sanguínea, se conoce como dislipidemia. Varios autores expresan que esta condición se produce por la ingesta excesiva de grasas, azúcares, alcohol y la herencia familiar. De manera similar, existe evidencia científica que sostiene que la dislipidemia puede aparecer como efecto secundario el consumo de medicamentos para la diabetes, el hipotiroidismo, el sobrepeso y la obesidad (Alvirde-García, 2016).

En este sentido, en América Latina, se presenta un panorama similar. Es así que el estudio CARMELA (Cardiovascular Risk Factor Multiple Evaluation in Latin America) (Pramparo, Boissonnet, \& Schargrodsky, 2011). Evaluó a 11.500 sujetos, hombres y mujeres entre 25 y 65 años, valorando los principales problemas asociados a enfermedades cardiovasculares en siete ciudades de América Latina. Este estudio determinó que las tasas de dislipidemia son mayores en los individuos de sexo masculino que en femeninos. Así tenemos que el 75,5\% de hombres y 48,7\% de mujeres en Barquisimeto (Venezuela), 70\% de hombres y 47,7\% de mujeres en Bogotá Colombia, 50,4\% de hombres y 24,1\% de mujeres en Buenos Aires (Argentina), 73,1\% de hombres y 62,8\% de mujeres en Lima (Perú), 62,5\% de hombres y el 37,5\% de mujeres, en la Ciudad de 


\section{Perfil lipídico como factor de riesgo cardiovascular en militares activos \\ Hospital General II-de Libertad 2019}

Vol. 3, núm. 3 Esp., (2019)

María Isabel Pacheco Arias; Holguer Estuardo Romero Urrea

México (México), el 52,2\% de hombres y el 38,1\% de mujeres, en Quito (Ecuador), y finalmente el 50,8\% de hombres y el 32,8\% de mujeres, en Santiago (Chile).

En este sentido, los gobiernos también luchan por reducir el consumo excesivo del alcohol, los índices de tabaquismo en la población y además, resaltan la influencia de la dieta diaria en el desarrollo de este tipo de enfermedades, esto lo ratifica el hecho de que, en los últimos cincuenta años, el análisis de la relación entre la alimentación y las ECV, representa uno de los campos más activos en los estudios de la epidemiología nutricional (Bernabeu-Mestre, Galiana Sánchez, \& López, 2017). 


\section{Perfil lipídico como factor de riesgo cardiovascular en militares activos Hospital General II-de Libertad 2019}

Vol. 3, núm. 3 Esp., (2019)

María Isabel Pacheco Arias; Holguer Estuardo Romero Urrea

Tabla 1. Factores de Riesgo Cardiovascular

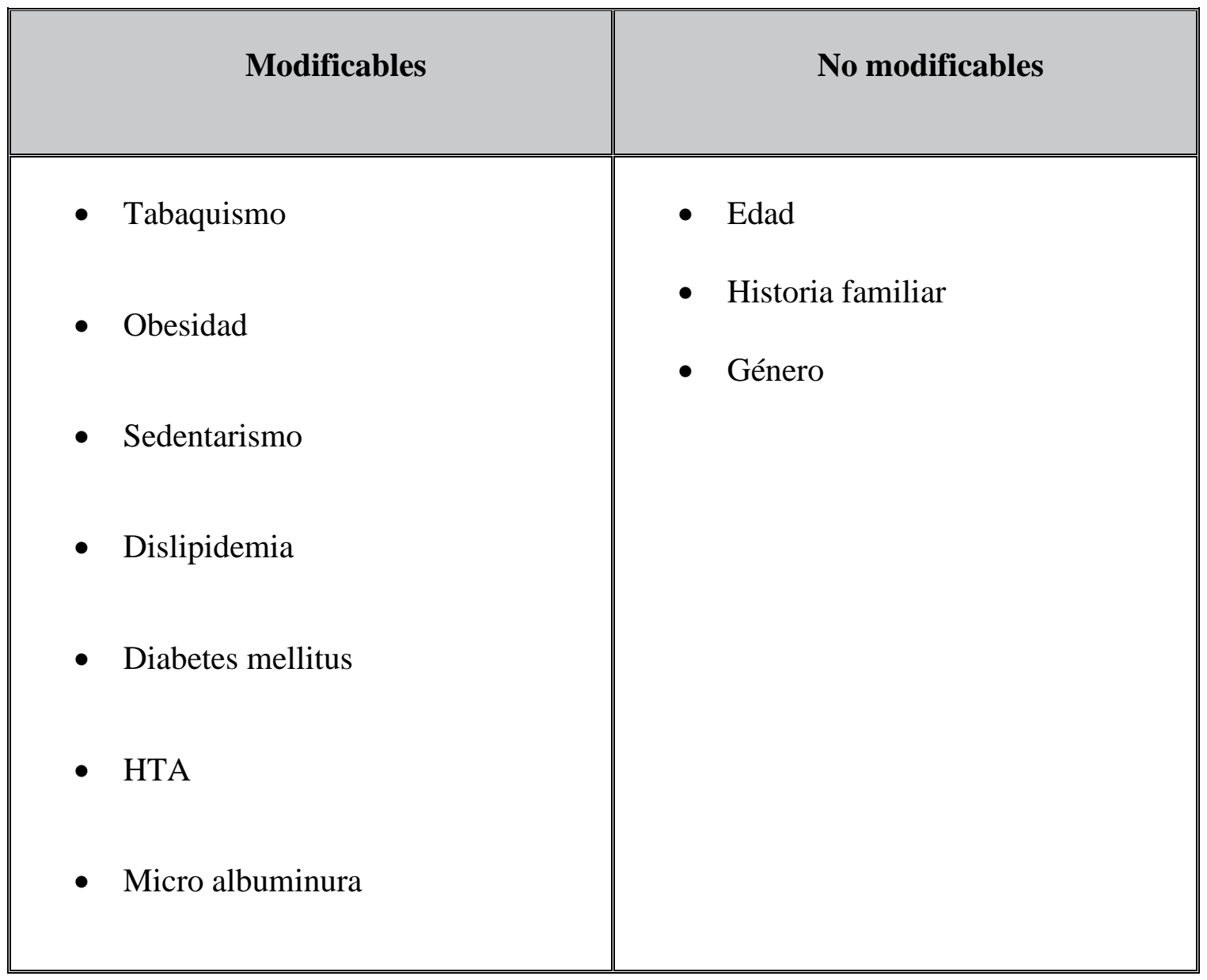

Fuente: (Cespedes \& Hu, 2015). Elaboración Propia.

Según datos del Ministerio de Salud Pública del Ecuador (MSPE), se estima que el 20\% de la población adulta tiene alteraciones de los niveles de colesterol, manteniendo niveles de colesterol superiores a los niveles normales. Además, la mayoría de estos, ya presentan dislipidemias. En este sentido, un estudio del MSPE, determinó que, durante el año 2015, el 52.4\% de la población padecía de sobrepeso, de éstos el 58.3\% son mujeres y 46,5\% son hombres. Así como también, el 15,3\% de la población total padece de obesidad, de ellos el 21.7 \% son mujeres 


\section{Perfil lipídico como factor de riesgo cardiovascular en militares activos \\ Hospital General II-de Libertad 2019}

Vol. 3, núm. 3 Esp., (2019)

María Isabel Pacheco Arias; Holguer Estuardo Romero Urrea

y el $8,9 \%$ son varones. Toda esta condición, incrementa substancialmente el riesgo de morbilidad por dislipidemias (Chalisquìn Cueva, 2016).

Las dislipidemias se clasifican en dislipidemias primarias y dislipidemias secundarias, Las primarias están constituidas por los casos en los que lo prevalecen trastornos caracterizados por defectos en las enzimas, receptores o metabolitos que participan en la síntesis y eliminación de las lipoproteínas, la más frecuente es la hipercolesterolemia familiar, seguida por hiperlipidemia familiar combinada, disbetalipoproteinemia e hipertrigliceridemia familiar. En el grupo secundario se incluyen alteraciones en los lípidos como consecuencia de otras enfermedades: diabetes mellitus, hipotiroidismo, síndrome nefrótico, uso de algunos fármacos (Cordero, Arévalo, Izquierdo, \& Torres, 2017).

Tabla 2. Tipos de dislipidemias según el perfil lipídico

\begin{tabular}{|c|l|}
\hline \multicolumn{1}{|c|}{ Tipo de dislipidemia } & \multicolumn{1}{|c|}{ Características } \\
\hline Hipercolesterolemia aislada: & $\begin{array}{l}\text { Aumento del colesterol total a expensas del } \\
\text { colesterol de las lipoproteínas de baja densidad (C- } \\
\text { LDL). }\end{array}$ \\
\hline Hipertrigliceridemia aislada & Aumento de los triglicéridos de origen endógeno \\
(a expensas de las lipoproteínas de muy baja
\end{tabular}




\section{Perfil lipídico como factor de riesgo cardiovascular en militares activos Hospital General II-de Libertad 2019}

Vol. 3, núm. 3 Esp., (2019)

María Isabel Pacheco Arias; Holguer Estuardo Romero Urrea

\begin{tabular}{|c|l|}
\hline & $\begin{array}{l}\text { densidad, VLDL), exógeno (a expensas de } \\
\text { quilomicrones), o ambos. }\end{array}$ \\
\hline Hiperlipemia mixta & Aumento del colesterol total y los triglicéridos. \\
\hline Hipoalfalipoproteinemia & alta densidad \\
& Disminución del colesterol de las lipoproteínas de \\
& (C-HDL \\
\hline
\end{tabular}

Fuente: Elaboración Propia.

La clasificación de las dislipidemias presentada, permite identificar el riesgo del paciente mediante aproximaciones. Esto es, si el paciente presenta un aumento de los niveles plasmáticos del CT, con incremento moderado de los Triglicéridos y una reducción del nivel de CHDL, este individuo, incrementará su riesgo de padecer algún accidente cardiovascular. Así mismo, si el paciente presenta una elevación severa de los triglicéridos, incrementará su riesgo de padecer una pancreatitis aguda. Esta clasificación orienta al médico en cuanto al tratamiento específico de la dislipidemia (Brites, Rosso, Meroño, Boero, \& Rivera, 2012).

\section{Metodología.}

Este trabajo de investigación, es de tipo cuantitativo, ya que considera las mediciones de los parámetros asociados al perfil lipídico, la tensión arterial, el índice de masa corporal y el nivel de glucosa en la sangre. Valores que serán tabulados a partir de la ficha médica de los militares 


\section{Perfil lipídico como factor de riesgo cardiovascular en militares activos Hospital General II-de Libertad 2019}

Vol. 3, núm. 3 Esp., (2019)

María Isabel Pacheco Arias; Holguer Estuardo Romero Urrea

activos, registrada en el Hospital general II DE Libertad. Además, esta investigación, es considerada un trabajo de cohorte trasversal con base exploratoria y tipo descriptivo, ya que, con los datos obtenidos de las fichas citadas, se describirán los niveles de los elementos del perfil lipídico de la población estudiada, y las variables asociadas a la prevalencia del riesgo cardiovascular.

Además, esta investigación, es considerada un trabajo de cohorte trasversal con base exploratoria y tipo descriptivo, ya que, con los datos obtenidos de las fichas citadas, se describirán los niveles de los elementos del perfil lipídico de la población estudiada, y las variables asociadas a la prevalencia del riesgo cardiovascular.

La población objetivo de esta investigación está conformada por todos los militares activos que asistieron a sus controles médicos anuales Hospital general II DE Libertad, en el Cantón Guayaquil. Se trata de 700 personas, que generaron un número similar de fichas médicas, durante su control médico rutinario, durante febrero y agosto del presente año.

Para la selección de los 248 individuos que integran la muestra, utilizamos el Muestreo Aleatorio Simpe, a partir del cual, generamos un listado de todos los militares activos (700) que acudieron al control médico periódico al Hospital general II DE Libertad, durante los meses de febrero a agosto del 2019.

En cuanto a la técnica utilizada para la medición de los parámetros poblacionales, se utiliza la ficha médica que es generada durante la revisión médica periódica a la que se someten los miembros de nuestra población objetivo. 
Perfil lipídico como factor de riesgo cardiovascular en militares activos Hospital General II-de Libertad 2019

Vol. 3, núm. 3 Esp., (2019)

María Isabel Pacheco Arias; Holguer Estuardo Romero Urrea

\section{Resultados.}

Tabla 3. Género de los encuestados

\begin{tabular}{|c|c|c|c|}
\hline \multicolumn{3}{|c|}{ Género } \\
\hline Categoría & Frecuencia & Porcentaje & $\begin{array}{c}\text { Porcentaje } \\
\text { Acumulado }\end{array}$ \\
\hline Masculino & 240 & $97 \%$ & $97 \%$ \\
\hline Femenino & 8 & $3 \%$ & $100 \%$ \\
\hline Total & 248 & $100 \%$ & \\
\hline
\end{tabular}

Fuente: Elaboración Propia

Del análisis de los datos recogidos mediante el Muestreo Aleatorio Simple, se desprende que el 97\% de los individuos investigados son Hombres y solo el 3\% son mujeres. 


\section{Perfil lipídico como factor de riesgo cardiovascular en militares activos \\ Hospital General II-de Libertad 2019}

Vol. 3, núm. 3 Esp., (2019)

María Isabel Pacheco Arias; Holguer Estuardo Romero Urrea

Imagen 1. Distribución agrupada de edad de los pacientes

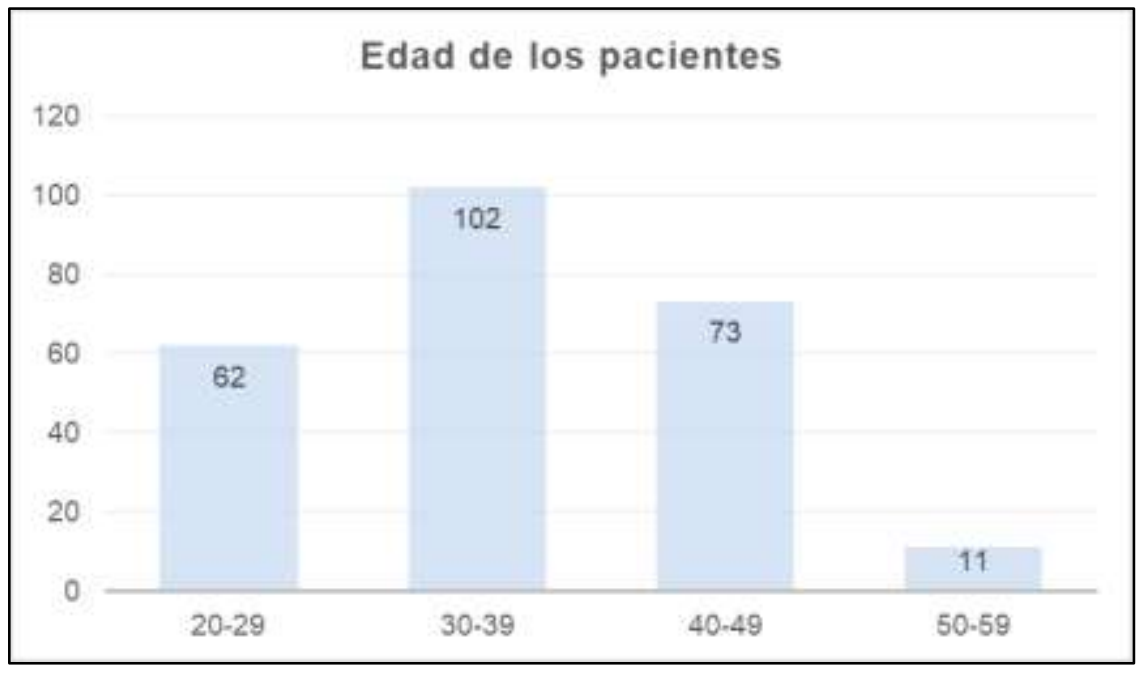

Fuente: Elaboración Propia.

A partir de este análisis preliminar, podemos resaltar que el promedio de edad de los individuos estudiados es de 36 años, el menor de los pacientes tiene 20 años y el mayor 56 años. Otro dato interesante que se desprende de este análisis es que, la mitad de los encuestados tiene 37 años o menos.

Índice de Masa Corporal (IMC) 


\section{Perfil lipídico como factor de riesgo cardiovascular en militares activos Hospital General II-de Libertad 2019}

Vol. 3, núm. 3 Esp., (2019)

María Isabel Pacheco Arias; Holguer Estuardo Romero Urrea

Imagen 2. Valores del IMC de la población estudiada

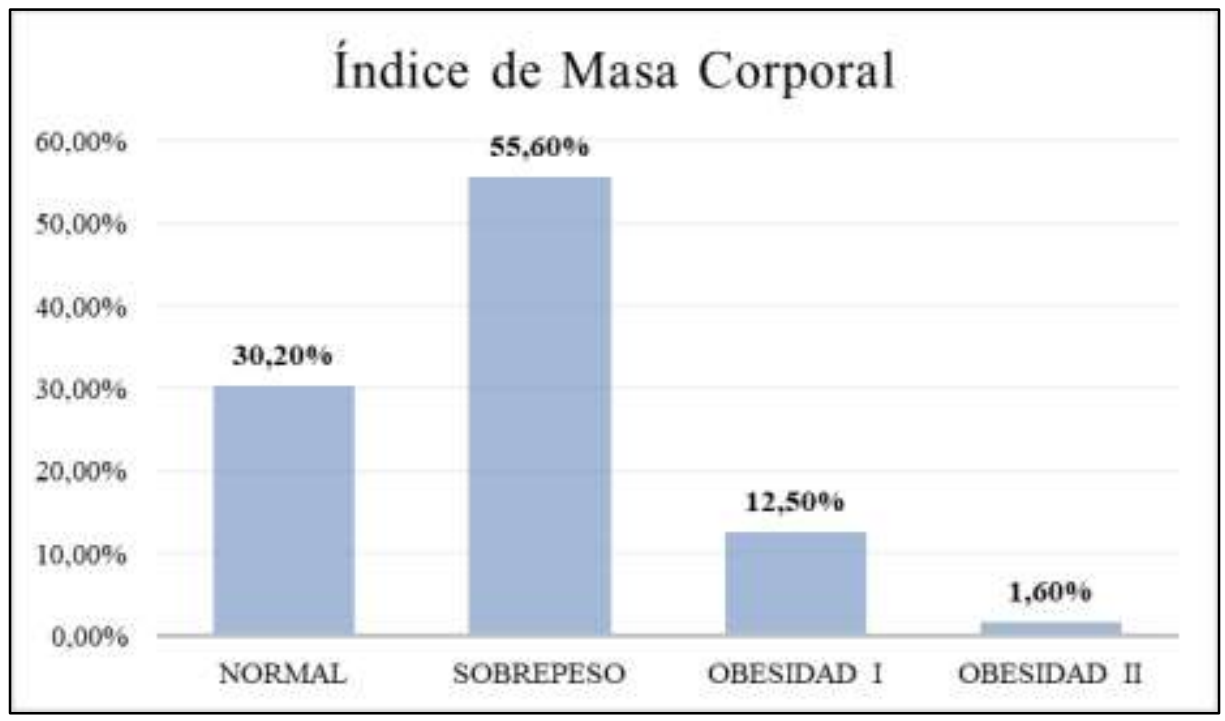

Fuente: Elaboración Propia.

Mediante la agrupación de datos, en función de los valores referenciales propuestos por la Organización Mundial de la Salud, se logró determinar que solo el 30,20\% de los pacientes tiene peso normal, lo que indica que cerca el $70 \%$ de ellos presenta sobrepeso y lo preocupante es que más del 14\% sufre de obesidad en algún grado. Estos datos los detallamos en la imagen 2. 
Perfil lipídico como factor de riesgo cardiovascular en militares activos Hospital General II-de Libertad 2019

Vol. 3, núm. 3 Esp., (2019)

María Isabel Pacheco Arias; Holguer Estuardo Romero Urrea

Tensión Arterial

Tabla 4. Estadística descriptiva de los valores de la Tensión Arterial de los investigados

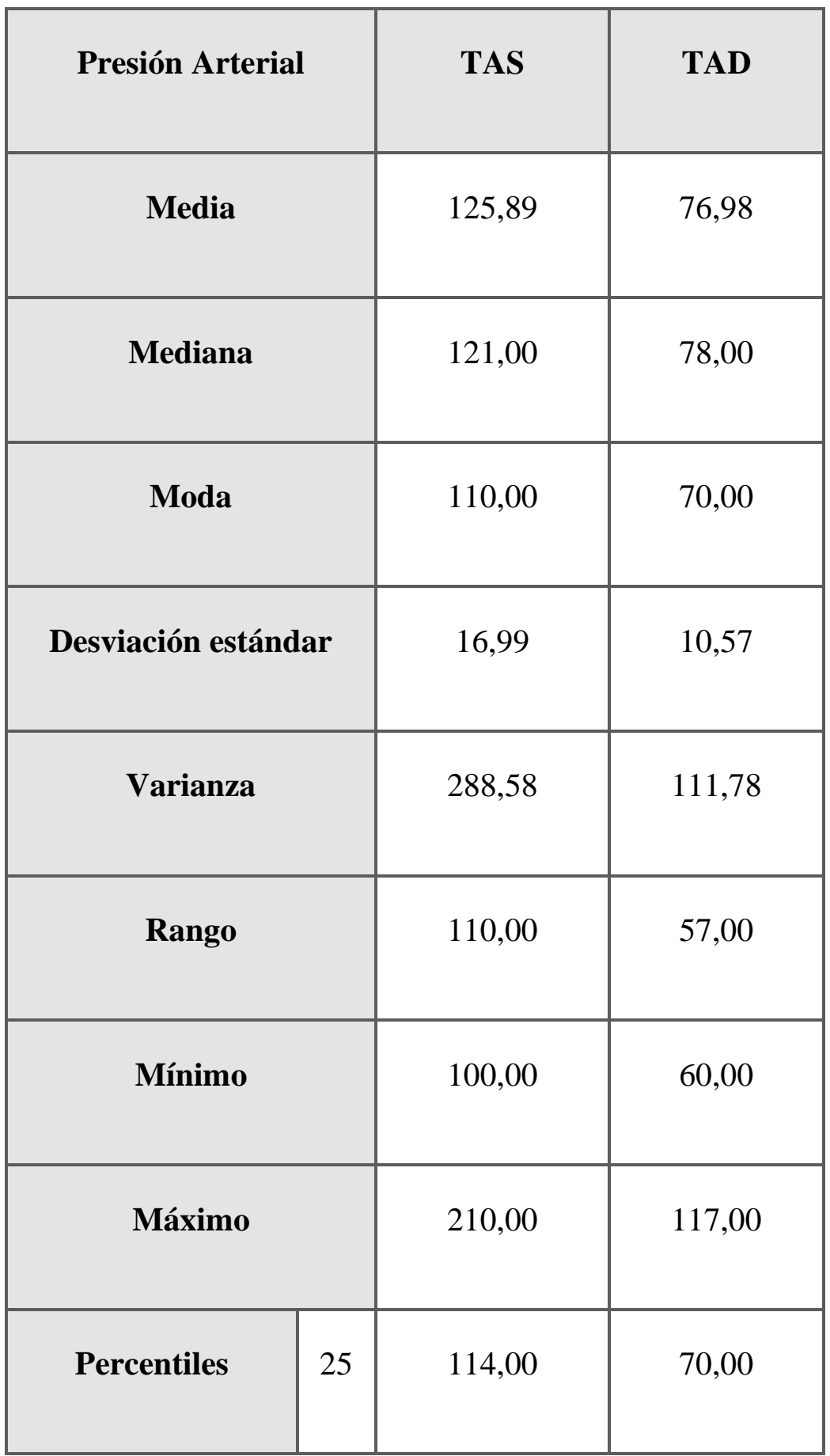




\section{Perfil lipídico como factor de riesgo cardiovascular en militares activos Hospital General II-de Libertad 2019}

Vol. 3, núm. 3 Esp., (2019)

María Isabel Pacheco Arias; Holguer Estuardo Romero Urrea

\begin{tabular}{|l|c|c|c|}
\hline & 50 & 121,00 & 78,00 \\
\hline & 75 & 135,75 & 80,00 \\
\hline
\end{tabular}

Fuente: Elaboración Propia.

En este sentido, la TA fue valorada durante el estudio clínico a todos los militares en este estudio. A partir de lo cual se determinó que la media de Tensión Arterial Sístole (TAS) fue de 125.89 mmHg y el valor medio de la Tensión Arterial Diástole (TAD) fue de 76,98 mmHg. Valores que podrían ser considerados normales, pero existen importantes variaciones en los datos recogidos, tales como el máximo de TAS llega a 210 mmHg y en el caso de la TAD, este se eleva sobrepasa los $111 \mathrm{mmHg}$.

Imagen 3. Distribución de los valores de la Tensión Arterial

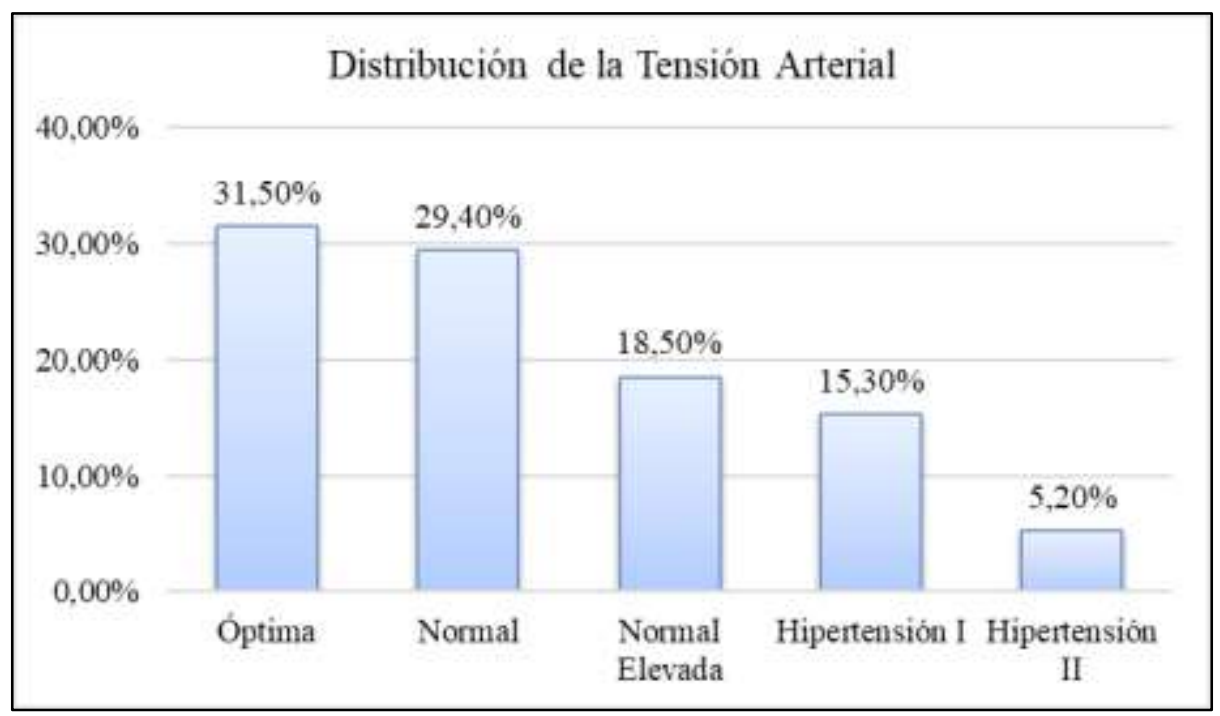

Fuente: Elaboración Propia. 


\section{Perfil lipídico como factor de riesgo cardiovascular en militares activos}

Hospital General II-de Libertad 2019

Vol. 3, núm. 3 Esp., (2019)

María Isabel Pacheco Arias; Holguer Estuardo Romero Urrea

Resulta preocupante que, de la población analizada, casi 4 de cada 10 pacientes presenta niveles elevados de Tensión Arterial. (Ver imagen 3), lo que incrementa su riesgo de padecer enfermedades cardiovasculares.

\section{Triglicéridos}

Tabla 5. Estadística descriptiva de los valores de triglicéridos en sangre

\begin{tabular}{|c|c|}
\hline Triglicéridos & Valores \\
\hline Media & 158,08 \\
\hline Mediana & 155,00 \\
\hline Moda & 170,00 \\
\hline Desviación estándar & 89,88 \\
\hline Varianza & $8.078,23$ \\
\hline Rango & \\
\hline
\end{tabular}




\section{Perfil lipídico como factor de riesgo cardiovascular en militares activos Hospital General II-de Libertad 2019}

Vol. 3, núm. 3 Esp., (2019)

María Isabel Pacheco Arias; Holguer Estuardo Romero Urrea

\begin{tabular}{|c|c|c|}
\hline \multicolumn{2}{|c|}{ Mínimo } & 19,90 \\
\hline \multicolumn{2}{|c|}{ Máximo } & 585,00 \\
\hline Percentiles & 25 & 89,00 \\
\hline & 50 & 155,00 \\
\hline & 75 & 190,00 \\
\hline
\end{tabular}

Fuente: Elaboración Propia.

De manera similar se detectaron valores mínimos por debajo de los parámetros normales, esto evidenció la existencia de 3 casos que presentan valores de Triglicéridos menores a 40 mg/dL. Y dos casos que superan los $500 \mathrm{mg} / \mathrm{dL}$, situándose en el nivel considerado Triglicérido muy alto, valores que, según varios autores incrementan el riesgo de sufrir accidentes cardiovasculares. 


\section{Perfil lipídico como factor de riesgo cardiovascular en militares activos \\ Hospital General II-de Libertad 2019}

Vol. 3, núm. 3 Esp., (2019)

María Isabel Pacheco Arias; Holguer Estuardo Romero Urrea

Imagen 4. Distribución del nivel de triglicéridos en sangre

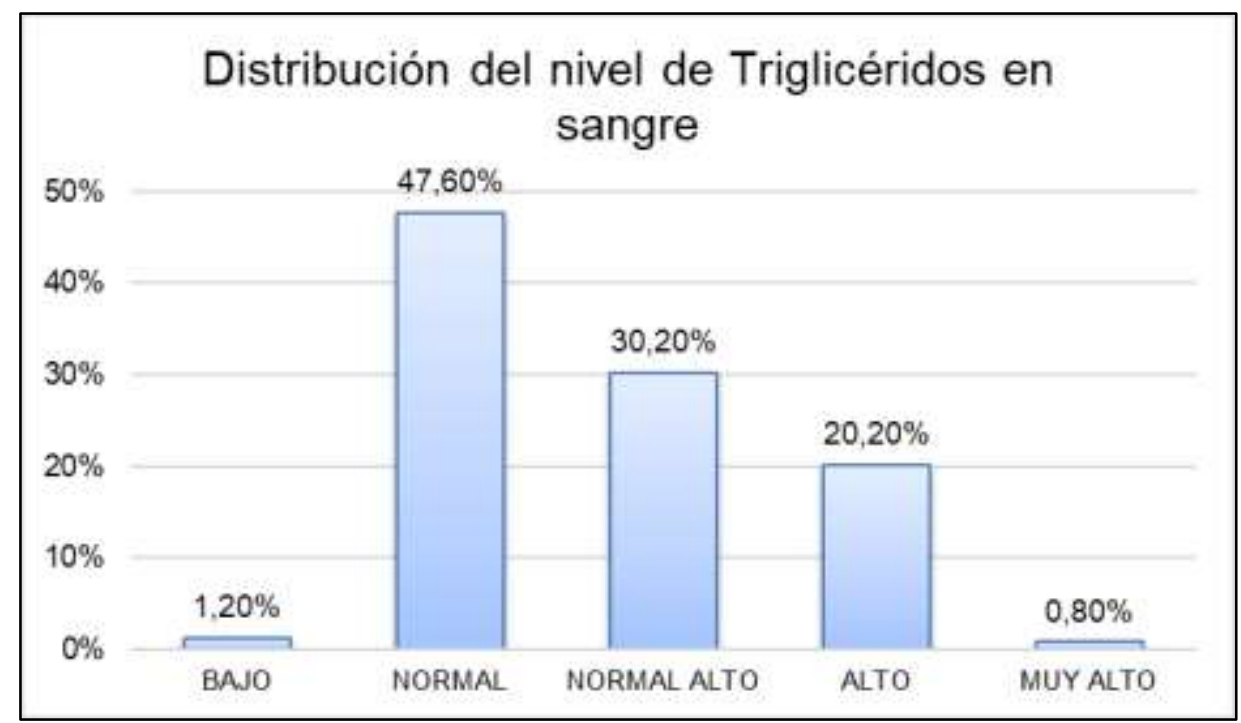

Fuente: Elaboración Propia.

En este sentido, se resalta que poco menos de la mitad, de los pacientes estudiados presenta un índice normal de Triglicéridos en sangre, mientras que alrededor poco más del 50\% de los estudiados, presenta alteraciones al alza del nivel de estos lípidos en la sangre. Lo que incide directamente con el riesgo de padecer enfermedades cardiovasculares e incluso la muerte del paciente.

\section{Colesterol Total en sangre}

En este sentido poco más del $32 \%$ de los pacientes investigados, presenta niveles elevados de colesterol, de éstos el 43\%, presenta niveles muy altos de colesterol en sangre. Estos niveles que sobrepasan los $240 \mathrm{mg} / \mathrm{dL}$, incrementan el riesgo de padecer accidentes cardiovasculares, y si se consideran los parámetros tratados con anterioridad, la población investigada se encuentra en 


\section{Perfil lipídico como factor de riesgo cardiovascular en militares activos Hospital General II-de Libertad 2019}

Vol. 3, núm. 3 Esp., (2019)

María Isabel Pacheco Arias; Holguer Estuardo Romero Urrea

riesgo cardiovascular. Pero estas inferencias las mostraremos más adelante. En la imagen 5, se muestra la distribución de los niveles de colesterol total en sangre de los pacientes investigados.

Imagen 5. Distribución del colesterol total en sangre

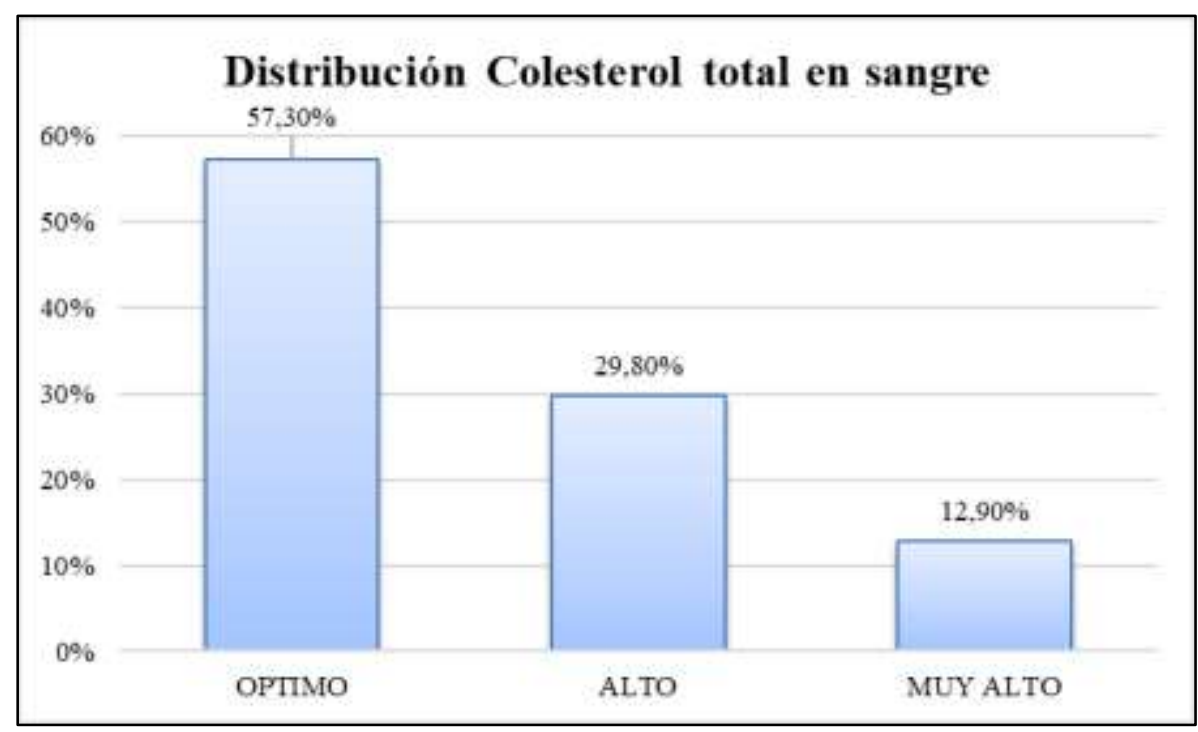

Fuente: Elaboración Propia.

HDL- Lipoproteína de Alta Densidad

Una vez categorizados los datos registrados en función de las tablas de referencia mundial para este tipo de colesterol, se logró diagnosticar de mejor manera el nivel de HDL, en los militares activos que participaron en este estudio. En este sentido, se considera oportuno mencionar que el $21 \%$ de los datos registrados, se encuentran por debajo de $40 \mathrm{mg} / \mathrm{dL}$, que es el valor mínimo recomendado por la Organización Mundial de la Salud. Por otro lado, cerca del $80 \%$ de los datos registrados, muestra valores de HDL normales y óptimos. 


\section{Perfil lipídico como factor de riesgo cardiovascular en militares activos \\ Hospital General II-de Libertad 2019}

Vol. 3, núm. 3 Esp., (2019)

María Isabel Pacheco Arias; Holguer Estuardo Romero Urrea

LDL - Lipoproteína de baja densidad

Poco más de 39\% de los pacientes estudiados, presenta niveles de LDL superiores a los valores óptimos recomendados por la OMS.

\section{Conclusiones.}

- Del análisis de los datos se desprende que gran parte de la población estudiada padece sobrepeso.

- Está claro que la mayoría de los militares estudiados, presenta alteraciones en el perfil lipídico, centrándose principalmente en niveles elevados de triglicéridos, colesterol total, colesterol HDL y bajos niveles de colesterol LDL.

- Otro elemento analizado es el nivel de tensión arterial, del que sabemos que cerca del $40 \%$ de la población estudiada presenta niveles elevados de TA.

- La distribución de la variable género, refleja la mínima participación de las mujeres en la vida militar, todo esto, porque hasta hace pocos años, las mujeres no eran admitidas en ningún tipo de programa de formación militar y, además en ciertos estratos sociales, existe rechazo a las mujeres que optan por esta profesión.

Recomendaciones

- Se recomienda aplicar la propuesta de educación nutricional y activar un conjunto de ejercicios activos que mejore los indicadores estudiados. 


\section{Perfil lipídico como factor de riesgo cardiovascular en militares activos Hospital General II-de Libertad 2019}

Vol. 3, núm. 3 Esp., (2019)

María Isabel Pacheco Arias; Holguer Estuardo Romero Urrea

- El estudio ha permitido determinar los valores asociados al perfil lipídico de la población estudiada, sería muy oportuno ejecutar un seguimiento a los militares seleccionados en la muestra, a través de un estudio longitudinal.

- Como investigación a futuro, se podría ampliar este trabajo, incluyendo un estudio del estilo de vida de los militares analizados, para definir de mejor manera el riesgo cardiovascular de los individuos estudiados.

\section{Bibliografía.}

Alvirde-García, U. (2016). Dislipidemias e hipertensión arterial. Gaceta medica de Mexico, 125(51), 56-62.

Bernabeu-Mestre, J., Galiana Sánchez, M., \& López, T. (2017). La gastronomía ante los retos epidemiológico-nutricionales del siglo XXI. Revista Española de Nutrición Humana y Dietética, 21(3), 209-212.

Brites, F. D., Rosso, L., Meroño, T., Boero, L., \& Rivera, S. (2012). Clasificación y diagnóstico bioquímico de las dislipemias. Síndrome Metabólico y Riesgo Vascular, 3(7), 150-166.

Cespedes, E. M., \& Hu, F. (Mayo de 2015). Dietary patterns: from nutritional epidemiologic analysis to national guidelines. Am J Clin Nutr, 101(5), 899-900.

Chalisquìn Cueva, J. (2016). Adherencia al tratamiento no farmacológico de dislipidemias en pacientes atendidos en el Hospital Provincial General Latacunga, enero-junio 2015. Ambato.

Cordero, S. P., Arévalo, C., Izquierdo, P., \& Torres, C. (2017). Prevalencia y factores asociados a la dislipidemia en los adultos de las parroquias urbanas de la ciudad de Cuenca, 2015-2016. Archivos Venezolanos de Farmacología y Terapéutica, 36(4), 101-105.

Estruch, R., \& Camafort, M. (2015). Dieta mediterránea y perfil lipídico plasmático. Revista Española de Cardiología, 68(4), 279-281.

Organización Mundial de la Salud. (17 de 05 de 2017). Enfermedades Cardiovasculares . Obtenido de Organización Mundial de la Salud: https://www.who.int/es/news-room/factsheets/detail/cardiovascular-diseases-(cvds) 


\section{Perfil lipídico como factor de riesgo cardiovascular en militares activos}

Hospital General II-de Libertad 2019

Vol. 3, núm. 3 Esp., (2019)

María Isabel Pacheco Arias; Holguer Estuardo Romero Urrea

Pramparo, P., Boissonnet, C., \& Schargrodsky, H. (2011). Evaluación del riesgo cardiovascular en siete ciudades de Latinoamérica: las principales conclusiones del estudio CARMELA y de los subestudios. Revista argentina de cardiología, 79(4), 377-382.

$$
\text { (c) (1) (5) (2) }
$$

RECONOCIMIENTO-NOCOMERCIAL-COMPARTIRIGUAL

CC BY-NC-SA

ESTA LICENCIA PERMITE A OTROS ENTREMEZCLAR, AJUSTAR Y CONSTRUIR A PARTIR DE SU OBRA CON FINES NO

COMERCIALES, SIEMPRE Y CUANDO LE RECONOZCAN LA AUTORÍA Y SUS NUEVAS CREACIONES ESTÉN BAJO UNA LICENCIA CON LOS MISMOS TÉRMINOS. 